\title{
Philosophiques
}

\section{Les loteries nationales : une exploitation malheureuse?}

\section{Pierre Desjardins}

Volume 12, numéro 1, printemps 1985

URI : https://id.erudit.org/iderudit/203279ar

DOI : https://doi.org/10.7202/203279ar

Aller au sommaire du numéro

Éditeur(s)

Société de philosophie du Québec

ISSN

0316-2923 (imprimé)

1492-1391 (numérique)

Découvrir la revue

Citer ce document

Desjardins, P. (1985). Les loteries nationales : une exploitation malheureuse? Philosophiques, 12(1), 191-196. https://doi.org/10.7202/203279ar d'utilisation que vous pouvez consulter en ligne.

https://apropos.erudit.org/fr/usagers/politique-dutilisation/ 


\section{INTERVENTION}

\section{LES LOTERIES NATIONALES : UNE EXPLOITATION MALHEUREUSE ?}

par Pierre Desjardins

L'étude du phénomène des loteries peut être un terrain fécond pour la critique de l'idéal d'une société et du genre de vie qu'elle propose à ses citoyens. Plus particulièrement au Québec, la place du marché des loteries a pris dernièrement une grande expansion. L'ensemble du volume des ventes connaît un essor fulgurant et, dans tout cela, l'État reste le principal bénéficiaire. Aussi je poserai deux questions essentielles :

1 - D'où les loteries, comme phénomène social contemporain des pays industrialisés, tiennent-elles cette grande popularité ?

2 - Est-ce néfaste pour la population, et, si oui, existe-til une panacée ou du moins, un remède?

Pour répondre à la première question, je voudrais, en me référant à Marcuse ${ }^{1}$, expliquer comment les loteries font partie intégrante du système socio-économique dans lequel nous évoluons. Posons la question : quelle est l'image du bonheur pour un citoyen d'aujourd'hui ? Vivre dans l'amour des autres ? . . . Aspirer à un monde de partage et d'équité ? En raison du genre de vie que la société technologique propose, peu voient encore là un sujet de préoccupation majeure ${ }^{2}$. Optimiser ses aptitudes personnelles pour parvenir à un développement culturel supérieur ? J'en doute, car avec l'avènement de la culture de masse,

1. Herbert Marcuse L'Homme Unidimensionnel, Éditions de Minuit, Paris, 1968, 312 pages.

2. Ibid., p. 277 à 300 . 
la dimension personnelle de la culture est presque disparue, c'est ce que Marcuse explique comme étant le phénomène d'assimilation culturelle des masses ${ }^{3}$. À moins que ce soit tout bonnement arriver au paradis à la fin de ses jours . . . Mais, à ce sujet, je vous rappelle qu'il y a déjà eu un pari de fait, un pari que Blaise Pascal s'est d'ailleurs empressé, je crois, de gagner . . ., du moins espérons-le pour lui. Non, soyons sérieux. L'idéal de la population est beaucoup moins idyllique que cela, et il se résume en un mot : " consommation ». Quoi de plus exaltant pour un citoyen de classe moyenne que de passer un après-midi de congé dans un grand centre commercial à s'acheter à peu près n'importe quoi, ou peut-être même rien du tout ?

Chez le citoyen de classe moyenne, l'état d'euphorie particulier qu'un après-midi de magasinage procure, s'explique en ce que, dans sa vie, cette expérience constraste avec l'ennui et la monotonie d'un travail banal et routinier exigé par le " devoirproduire " oppressant des sociétés technologiques, un " devoirproduire " absolu à travers lequel peu ou pas d'individus peuvent trouver une valorisation personnelle. Et c'est en ce sens que la possession soudaine d'un ou de plusieurs millions de dollars, par le truchement d'un boulier, fait des citoyens heureux, parce qu'aptes au bonheur total : le bonheur qu'ils auront de pouvoir enfin se payer une surconsommation à l'infini. Mais attention, l'investissement d'un ou cinq dollars dans une loterie ne signifie pas nécessairement pour les consommateurs qu'ils ont l'illusoire certitude de gagner le gros lot : ce que ce billet de loterie leur procure, à travers le rêve, c'est la sensation passionnée de participer au monde du " plaisir de la consommation ", et il leur donne surtout l'assurance et le réconfort du " tu peux encore " nécessaire pour affronter l'ennuyeuse platitude de leur vie monotone de petits travailleurs.

Nous retrouvons le même phénomène dans le système des cartes de crédit : quoi de plus valorisant pour un citoyen ordinaire que d'en exhiber toute une ribambelle? Ici encore, c'est l'accroissement du niveau virtuel d'achat qu'elle offre à son détenteur qui est significatif, car il faut comprendre que, lorsque dans une société de production comme la nôtre, la satisfaction

3. Ibid., p. 89 à 105. 
des besoins matériels devient prétexte à la valorisation des individus, la surmultiplication de ces besoins et leur satisfaction, même imaginaire, devient essentielle pour maintenir en place les idéaux proposés. Si la société a transformé le citoyen en consommateur, nous n'avons donc pas à nous surprendre, je crois, qu'il aspire à son pendant le plus élevé, la surconsommation, et c'est précisément cette tendance que lui permet d'actualiser mentalement un billet de loterie.

Il est d'ailleurs amusant de remarquer que, d'un point de vue psychologique, le parieur considère inconsciemment, qu'en achetant un billet, il a autant de chances de gagner le gros lot que de ne pas le gagner . . . Car, de la même façon que celui qui magasine a l'excitante impression que toute cette marchandise est presque à lui parce qu'à sa portée, celui qui parie a déjà le sentiment que le gros lot est à lui, du moins jusqu'au prochain tirage. Cette sorte de jouissance individuelle justifie à elle seule l'achat du billet; ce qui motive alors, c'est la participation à un enjeu, un enjeu socio-culturel qui dépasse de beaucoup le simple enjeu matériel du montant en argent du gros lot.

Du reste, il serait aussi intéressant de noter que cette facilité pour chaque citoyen, pauvre ou riche, d'acheter un billet de loterie, et partant de devenir millionnaire, est une des tentatives les plus réussies du système actuel de réduire artificiellement les différences de classes : cela tient à la possibilité d'identification de "monsieur tout le monde " au gagnant, puisque son seul mérite, en somme, se résume à être tombé sur les "bons" numéros, ce qui peut finalement arriver à n'importe qui, quel que soit son rang social, et ne relève d'aucune capacité personnelle particulière. C'est ce phénomène d'identification qui explique par exemple la réaction caractéristique du parieur lorsque, au tirage, tout excité, il s'efforce de trouver une ressemblance quelconque, même fort lointaine, entre sa combinaison perdante et celle du gagnant ; fièrement, il constatera probablement qu'il n'était pas si loin que ça de l'emporter aussi, ce qui pour lui constitue un gage d'avenir et un signe du ciel qu'il faut encore recommencer ; et, s'il a l'heureux privilège de remporter un dix ou un vingt-cinq dollars, ce sera pour lui une gratification sans précédent et tout un honneur, et il se fera un devoir de réinvestir cette somme dans les loteries. 
Ayant établi que les loteries, parce que correspondant aux nouveaux modèles établis, constituent un mal nécessaire du système en place, et cela, bien avant son appropriation par l'État, on peut aborder maintenant la deuxième question : peut-on remédier à la situation, et, si oui, de quelle façon ? Avant de répondre, je ferai d'abord une remarque : dans leur développement, les modes de production ne sont plus aujourd'hui guidés directement par l'État, mais bien par les grandes sociétés productrices, et, en ce sens, je ne crois pas que l'État puisse s'intercaler directement entre ce qui est devenu ce désir incontrôlé de nouveaux besoins du citoyen et les exigences économiques de ces dites sociétés, et cela même si ces besoins sont la plupart du temps "faux " et vont à l'encontre du développement des individus. On voit mal par exemple un gouvernement proposer une réduction de la consommation : rappelons-nous toute l'impopularité des "mesures Trudeau ", mesures qui pourtant ne se voulaient que transitoires. En ce sens, l'interdiction pure et simple des loteries par l'État, mesure qui, à prime abord, peut sembler la plus simple à prendre, reste dans les faits irréaliste et dangereuse, le monde interlope s'accaparant alors allègrement, à la place de l'État, de ce marché tout cuit d'avance.

Cette remarque faite, il demeure cependant très pertinent de se demander si l'État a le droit de promouvoir exagérément ce marché, au détriment d'une qualité de vie qui en est déjà passablement affectée, et cela, dans le seul but de garnir ses coffres. Car, au train où vont les choses actuellement, on pourrait presque qualifier le gouvernement de "marchand de rêves". C'est du moins l'image qu'il projette selon moi à travers sa gérance de Loto-Québec, une entreprise d'état qui fonctionne exactement comme une entreprise privée, c'est-à-dire en maximisant ses ventes sans se soucier des conséquences socio-culturelles et en utilisant toute la quincaillerie habituelle d'une entreprise privée : marketing, publicité, diversification de la production. C'est un des organismes d'État qui fonctionne le mieux actuellement au Québec : il a réussi à mettre sur le marché un produit beau, attrayant, coloré, entièrement informatisé, à la portée de toutes les bourses, et sous cinq marques différentes (huit si l'on considère les loteries inter-provinciales). Qu'est-ce qu'un consommateur peut espérer de mieux ?... Sa marque de 
bière ? . . . Oui, sans doute, car, disons-le tout de suite, du simple point de vue des probalités mathématiques, la loterie nationale reste pour le consommateur un bien mauvais achat : la somme des gains possibles pour les parieurs n'égale même pas la moitié du total des sommes qu'ils misent, le gouvernement s'arrogeant ici goulûment un $40 \%$ de profit net (en plus des frais d'exploitation de $15 \%$ ), un taux qui se rapproche beaucoup plus du taux d'un usurier que de celui d'un bon administrateur de compagnie. $\grave{A}$ ce propos, rappelons-nous qu'en 1976, le maire Drapeau avait au moins eu l'honnêteté d'identifier clairement son produit : « taxe volontaire olympique "; aujourd'hui tout est mis en œuvre pour masquer cette réalité.

L'utilisation de la publicité par Loto-Québec à cet égard m'apparaît particulièrement odieuse. Rappelons que dans une société comme la nôtre, ce ne sont pas tellement les besoins réels qui justifient la production de nouveaux produits, mais plutôt les besoins que l'on crée souvent de toutes pièces par le biais de la publicité ; ainsi, au moment où le nouveau produit fait son apparition sur le marché, le consommateur a déjà été conditionné à son besoins par la publicité. Qu'une entreprise privée utilise ce moyen me semble, dans le contexte actuel, " normal ", pour survivre et s'assurer une part du marché ; mais, est-il acceptable qu'une entreprise d'État comme Loto-Québec, qui détient le monopole des jeux de hasard (c'est-à-dire le monopole sur un produit dont le besoin est déjà inhérent au comportement même des citoyens), utilise le même procédé ? Mettons-nous du côté d'un consommateur moyen : qu'est-ce, pour lui, que dix dollars investis en loteries par semaine ? Cela peut représenter $2 \%$ de son salaire ; avez-vous cependant déjà calculé ce que cela peut représenter pour celui qui est au salaire minimum ? Plus de 6\%, ce qui est déjà une proportion considérable de son budget. Maintenant, par une publicité massive, on va réussir à doubler ou à tripler ce pourcentage, en faisant miroiter, aux yeux des gens mal nantis, un monde de plaisir et d'aisance, un monde auquel ceux qui sont démunis n'ont de toute façon aucun autre accès possible que celui ménagé par le gain magique . . . Voilà, à mon avis, ce qui frise l'indécence pour un gouvernement qui se veut respectueux de tous ses citoyens ... 
Et ne nous y trompons point : ce n'est pas, comme l'a suggéré Rueben Baetz des loteries ontariennes, en réduisant le montant des gros lots et en augmentant le nombre des lots secondaires, qu'on va régler ce problème. Au contraire. Si mille ou cinq mille dollars ne sont pas attrayants pour certains, ils le sont pour d'autres moins fortunés ; ainsi, on ne fera qu'aggraver cette situation : les plus riches seront moins intéressés devant des lots aux montants moins élevés, même s'ils sont plus nombreux, tandis que les moins riches augmenteront leur engouement devant des sommes qui, même moindres, les illusionnent encore.

Et je conclus en disant que, même si le phénomène des loteries ne peut être enrayé de façon draconienne, parce que constitutif d'un système économique dont le contrôle nous échappe, l'État a quand même la possibilité et le devoir de modérer sa croissance, en limitant volontairement l'expansion de son marché ; car il n'a pas le droit de boucler son budget en exploitant à fond les vices d'un système sans tenir compte du mieux-être aussi bien spirituel que matériel de ses citoyens. En ce sens, je crois que c'est toute sa politique des loteries qu'il urge de revoir, car l'enjeu socio-culturel est important.

Département de philosophie

Collège Montmorency. 